\title{
Functional Expression of Aquaporin-2 Tagged with Photoconvertible Fluorescent Protein in mpkCCD Cells
}

\author{
Kay-Pong Yip ${ }^{a}$ Byeong J. Cha ${ }^{a}$ Chung-Ming Tse ${ }^{b}$ Mateus E. Amin ${ }^{a}$ Jahanshah Amin ${ }^{a}$
}

\begin{abstract}
aDepartment of Molecular Pharmacology and Physiology, University of South Florida, Tampa, Florida, bDepartment of Medicine, GI Division, Johns Hopkins University School of Medicine, Baltimore, Maryland, USA
\end{abstract}

\section{Key Words}

Aquaporin-2 - Photoconvertible fluorescent protein

\begin{abstract}
Background: Vasopressin induced trafficking of aquaporin-2 (AQP2) containing vesicles has been studied in kidney cell lines using conventional fluorescent proteins as tags. However, trafficking of fluorescent tagged AQP2, which resembles the vectorial translocation of native AQP2 from cytoplasm to apical membrane has not been demonstrated at real time. Using a photoconvertible fluorescent protein tag on AQP2 might allow the simultaneous tracking of two separate populations of AQP2 vesicle after subcellular local photoconversion. Methods: A spacer was used to link a photoconvertible fluorescent protein (mEos2) to the aminoterminus of AQP2. The DNA constructs were expressed in mpkCCD cells. The trafficking of chimeric protein was visualized with high speed confocal microscopy in 4 dimensions. Results: Chimeric AQP2 expressed in mpkCCD cell conferred osmotic water permeability to the cells. Subcellular photoconversion with a $405 \mathrm{~nm}$ laser pulse converted green chimeras to red chimeras locally. Forskolin stimulation triggered chimeric AQP2 to translocate from acidic organelles to apical plasma membrane. By serendipity, the rate of apical accumulation was found to increase when mEos2 was tagged to the carboxyl-terminus in at least one of the AQP2 molecules within the tetramer. Conclusion: Functional photoconvertible chimeric AQP2 was successfully expressed in mpkCCD cells, in which forskolin induced apical trafficking and accumulation of chimeric AQP2. The proof-of-concept to monitor two populations of AQP2 vesicle simultaneously was demonstrated.
\end{abstract}




\section{Cellular Physiology Cell Physiol Biochem 2015;36:670-682 \begin{tabular}{l|l}
\hline DOI: 10.1159/000430129 & (c) 2015 S. Karger AG, Basel
\end{tabular} \begin{tabular}{l|l} 
and Biochemistry Published online: May 21, 2015 & www.karger.com/cpb
\end{tabular} \\ Yip et al.: Expression of Aquaporin-2 Tagged with a Photoconvertible Protein}

\section{Introduction}

Vasopressin increases osmotic water permeability in principal cells of the kidney collecting duct by binding to the basolateral vasopressin type 2 receptors, which activates adenylyl cyclases [1] and increases cAMP level of the cells. cAMP then induced PKAdependent phosphorylation of aquaporin-2 (AQP2), translocation of AQP2, and fusion of AQP2 containing vesicles to the apical membrane [2-4]. Protein labeling is one of the most popular applications of fluorescent protein, allowing visualization of protein expression, localization, and translocation in living cells. AQP2 tagged with green fluorescent protein (GFP) or red fluorescent protein (DsRed) has been used to study AQP2 trafficking in LLC$\mathrm{PK}_{1}$ cells and MDCK cells $[5,6]$. However, trafficking of fluorescently-tagged AQP2, which resembles the vectorial translocation of endogenous AQP2 to apical membrane as found in the collecting duct, has not been demonstrated at real time.

Photoconvertible fluorescent proteins are a class of fluorescent proteins whose fluorescence properties can be switched by a pulse of light at a specific wavelength [7]. Fluorescent proteins undergoing green to red photoconversion have been used for the investigation of dynamic processes in living cells and for photo-localization microscopy $[8,9]$. mEos2 is a monomeric photoconvertible fluorescent protein whose emission can be switched from green to red by irradiation at a near-ultraviolet wavelength [10]. Subcellular local photoconversion of green-mEos 2 to red-mEos2 would potentially allow the simultaneous monitoring of two discrete subpopulations of the tagged protein at two spectral windows [9].

To evaluate the feasibility of using mEos 2 as a fluorescent protein tag to visualize vectorial translocation and apical accumulation of AQP2 at real time, mouse AQP2 was tagged with mEos2 at either the amino (N)- or carboxyl (C)-terminal position and the resulting chimeras, individually or in combination, were expressed in mpkCCD cells in vasopressin-free culture medium. mpkCCD cell is the cell model used for proteomic and phosphoproteomic studies of vasopressin signaling in collecting duct [11-14]. Forskolin-induced trafficking of the chimeric proteins was monitored with high speed confocal fluorescence microscopy in four dimensions (XYZ-T). Subcellular local photoconversion was induced with a $405 \mathrm{~nm}$ laser. The present study sought to provide the proof-of-concept to simultaneously monitor two populations of AQP2-containing vesicle, and to demonstrate the apical accumulation of mEos2-tagged AQP2 in mpkCCD cells at real time.

\section{Materials and Methods}

\section{Chimeric constructs of mEos2 tagged AQP2}

The mammalian expression vector EX-Mm01263-MO2 (Genecopeia) was used to drive the expression of chimeric proteins. The vector contains mouse AQP2 cDNA under the control of cytomegalovirus (CMV) promoter. To construct the chimeras, the mEos2 was amplified from pRSETa mEos2 plasmid DNA (Addgene) by polymerase chain reaction (PCR) using four pairs of oligonucleotides with each pair containing engineered DNA sequences encoding spacer proteins and restriction endonucleases. Spacer protein between the AQP2 and mEos2 of 19 amino acid long made of mainly alanines and glutamines (QQQQQAAAPAQQQQQEFGT) was engineered to allow optimization for AQP2 homo-oligomerization and potential trafficking. Following completion of PCR, the resulting DNA fragment was cleaved by the restriction enzymes and cloned into the EX-Mm01263-M02. These constructs express mEso2 in frame with a 19 amino acid long spacer at the amino-terminus of AQP2 (mEos2-AQP2) or carboxyl-terminus of AQP2 (AQP2-mEos2).

\section{Cell culture}

Experiments were performed on a mouse CCD principal cell line ( $\mathrm{mpkCCD}_{\mathrm{C} 14}$ cells) grown on permeable filters until polarization in vasopressin-free culture medium (Transwell, $0.4 \mu \mathrm{m}$ pore size). Endogenous AQP2 is not detectable when mpkCCD cells are grown in the absence of vasopressin $[15,16]$. Cells were maintained in a 1:1 mixture of DMEM/Ham's F12 medium, supplemented with dexamethasone (50 nM), 


\section{Cellular Physiology Cell Physiol Biochem 2015;36:670-682 \begin{tabular}{c|c}
\hline DOI: 10.1159/000430129 & (c) 2015 S. Karger AG, Basel
\end{tabular} \begin{tabular}{l|l} 
and Biochemistry Published online: May 21, 2015 & www.karger.com/cpb
\end{tabular}}

Yip et al.: Expression of Aquaporin-2 Tagged with a Photoconvertible Protein

triiodothyronine (1nM), selenium (60nM), insulin (5 $\mu \mathrm{g} / \mathrm{ml})$, mouse EGF (10ng/ml), transferrin $(5 \mu \mathrm{g} / \mathrm{ml})$, and $2 \%$ fetal calf serum in a humidified atmosphere with $5 \% \mathrm{CO}_{2}$ at $37^{\circ} \mathrm{C}$. Cells were seeded at $8 \times 10^{4}$ cells $/ \mathrm{cm}^{2}$ for transfection ( $0.5 \mu \mathrm{g}$ DNA $/ 1 \times 10^{5}$ cells). Next day, cells were transfected with Metafectene Pro transfection reagent (Biontex) for 24 hours according to manufacturer's instruction and selected with 800 $\mu \mathrm{g} / \mathrm{ml}$ Geneticin. Studies were performed in transfected cells from passages 1-4 after the initial transfection. For high-speed confocal fluorescent image collection, transfected mpkCCD cells were seeded on collagen coated glass bottom dishes (MatTek) and grown to confluence [17].

Measurement of osmotic water permeability mpkCCD cells

All experiments were performed at $37^{\circ} \mathrm{C}$ on confluent cells seeded on permeable filters and grown until polarization. Cells were bathed in 290 mOsm buffer solution on both side of the filter, and loaded with calcein-AM ( $5 \mu \mathrm{M}$, Invitrogen) for $15 \mathrm{~min}$ from the apical side. After washing, the solution on the apical side was then rapidly switched to $150 \mathrm{mOsm}$ buffer solution. The decrease of calcein fluorescence due to cell volume increase was monitored with a Leica SP5 confocal imaging unit mounted on an inverted microscope (excitation $488 \mathrm{~nm}$, emission 515-525 nm). Single cell fluorescence was measured with the Leica SP5 quantitative tool. After background subtraction, calcein fluorescence versus time was fitted to a single exponential function to give the exponential time constant $(\tau)$. The time constant was used as an index of osmotic water permeability $[16,18,19]$.

Immunofluorescence microscopy

mpkCCD cells grown on permeable filters were fixed with 4\% paraformaldehyde in PBS for 20 min in room temperature and washed with $50 \mathrm{mM} \mathrm{NH}_{4} \mathrm{Cl}$ in PBS. The cells were then permeabilized with $0.1 \%$ Triton-X100 in PBS for 10 min, treated with 0.5\% SDS-PBS for $5 \mathrm{~min}$, and blocked by 1\% BSA-PBS for 30 min in room temperature. The cells were then incubated with specific primary antibodies overnight at $4^{\circ} \mathrm{C}$, followed by incubation of corresponding secondary antibodies conjugated with DyLight 549 or DyLight 647 (3 $\mu \mathrm{g} / \mathrm{ml}$, Jackson ImmunoResearch) for 2 hours. Confocal fluorescence images were collected with a Leica SP5 laser scanning confocal microscope. Primary antibodies used for immunolocalization are AQP2

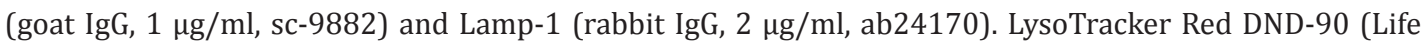
Technologies) was used to label acidic vesicular compartments in live mpkCCD cells grown on collagen coated glass bottom dishes.

Time-lapse imaging and quantification of apical accumulation of mEos2 fluorescence

Transfected mpkCCD cells were grown to confluence on glass bottom culture dishes for high speed 4D ( XYZ-T) confocal fluorescence image acquisition. 4D images of AQP2 fusion protein were acquired with an Olympus IX81 inverted microscope equipped with 3i spinning disk confocal system (Intelligent Imaging Innovations). The dish was placed in a stage-top environmental chamber (Pathology Device) that maintains temperature at $37^{\circ} \mathrm{C}$ and $5 \% \mathrm{CO}_{2}$ level. Translocation of AQP2 fusion protein in $4 \mathrm{D}$ was monitored with Olympus UPLSAPO $60 \mathrm{x}$ water immersion objective $(1.20 \mathrm{NA}$ ) by taking z series optical sections ( $1 \mu \mathrm{m}$ step size $\mathrm{x} 12$ sections) every $10 \mathrm{~min}$. In order to minimize the focus drift during imaging periods, the Olympus ZDC2 function was used and the temperature of objective was maintained at $37^{\circ} \mathrm{C}$ by a temperaturecontrolled sleeve (Pathology Device). The fluorescence signal from the fusion protein was captured with Evolve EMCCD (Photometrics) with a setting of 2x2 binning, $50 \mathrm{~ms}$ exposure, and intensification mode. The accumulation of AQP2 fusion proteins at the apical region of the cells was estimated during the recording period by measuring the mean intensity of a spherical volume $\left(\sim 45 \mu \mathrm{m}^{3}\right)$ after constructing 3D images by Vaa3d program $[20,21]$. The mean intensities were then normalized by the mean intensity of the first 3D image (time $=0$ ) in each time series after background subtraction.

\section{Photoconversion of mEos2}

Photoconversion was induced by a $405 \mathrm{~nm}$ laser irradiation $(0.5 \mathrm{~s})$ with the spinning disk confocal system. The area of irradiation, duration, and laser intensity were controlled via the operation system of spinning disk confocal system (SlideBook 5). Green mEos2 signal was collected with a 525/50 nm emission filter with $488 \mathrm{~nm}$ excitation. Red mEos 2 signal was collected with a 617/73 emission filter with $561 \mathrm{~nm}$ excitation. 


\section{Cellular Physiology Cell Physiol Biochem 2015;36:670-682 \begin{tabular}{|l|l|}
\hline DOI: 10.1159/000430129 & O 2015 S. Karger AG, Basel \\
\hline
\end{tabular} and Biochemistry Published online: May 21, $2015 \quad$ www.karger.com/cpb \\ Yip et al.: Expression of Aquaporin-2 Tagged with a Photoconvertible Protein}

\section{Solutions and Chemicals}

The isotonic solution (290 mOsm) used for measuring osmotic water permeability in mpkCCD cell contains (in mM): $\mathrm{NaCl}, 120 ; \mathrm{NaHCO}_{3}, 25 ; \mathrm{K}_{2} \mathrm{HPO}_{4}, 2 ; \mathrm{MgSO}_{4}, 1.2 ; \mathrm{CaCl}_{2}, 2$; glucose, 5.5; and sodium acetate, 5. The hypotonic solution (150 mOsm) was made by removing $76 \mathrm{mM} \mathrm{NaCl}$ from the isotonic solution. All solutions were gassed with $95 \% \mathrm{O}_{2}$ and $5 \% \mathrm{CO}_{2}$ before use, and $\mathrm{pH}$ was adjusted to 7.4. LysoTracker Red DND-99 was purchased from Life Technologies. AVP was purchased from Bachem. Forskolin and Geneticin were purchased from Sigma-Aldrich.

\section{Statistical Analysis}

Results were reported as mean \pm standard error of the mean. The exponential time constant $(\tau)$ was calculated using a nonlinear least-squares fitting program (Graphpad PRISM, GraphPad Software). BMDP statistical package Program 1R (Statistical Solution) was used for regression analysis and analysis of variance of regression coefficients.

\section{Results}

Expression of mEos2-AQP2 and AQP2-mEos2 chimeric proteins in mpkCCD cells

The expression of mEos2-AQP2 and AQP2-mEos2 fusion proteins in transfected mpkCCD cells were verified with anti-AQP2 antibody. Immunofluorescence of AQP2 was substantially overlapped with mEos2 fluorescence in cells transfected with mEos2-AQP2 DNA constructs (Fig. 1A and 1B). The Pearson's correlation coefficient is $0.63 \pm 0.03$ (5 series of xyz images from five filters), which indicated that the mEos2 fluorescence and AQP2 immunofluorescence are derived from the mEos2-AQP2 chimeric protein. mEos2-AQP2 was found primarily in vesicular structures scattered in the cytoplasm (see "Localization of mEos2-AQP2 in intracellular acidic compartments" section below). On the other hand, mpkCCD cells transfected with DNA construct of mEos2 alone exhibited distribution of mEos2 throughout the cytoplasm (Fig. 2A). Therefore, the inherent fluorescence of the mEos2-AQP2 chimera was appropriate as a localization marker of AQP2 in mpkCCD cells. Immunofluorescence of AQP2 was also overlapped with mEos2 fluorescence in cells transfected with AQP2-mEos2 DNA constructs (Fig. 1C and 1D). Pearson's correlation coefficient is $0.74 \pm 0.02$ ( 5 series of xyz images from five filters), indicating that mEos2 fluorescence and AQP2 immunofluorescence are derived from the AQP2-mEos2 chimeric protein. In contrast to $\mathrm{mEos} 2-\mathrm{AQP} 2$ fusion protein, $\mathrm{AQP} 2-\mathrm{mE}$ os 2 fusion protein expression was highly enriched in the apical plasma membrane even under non-stimulated condition (Fig. 2B). A similar expression pattern has also been demonstrated previously for a GFP tagged to AQP2 carboxyl terminus in LLC-PK 1 cells [5].

Apical osmotic water permeability of mpkCCD cells transfected with mEos2-AQP2 or AQP2-mEos2 constructs

Apical osmotic water permeability of mpkCCD cells was estimated by monitoring the rate of calcein fluorescence quenching when the apical solution was switched from a $290 \mathrm{mOsm}$ isotonic solution to a $150 \mathrm{mOsm}$ hypotonic solution. It has been shown that the expression of AQP2 in mpkCCD cells is not detectable when grown in vasopressin-free medium [15, 16]. Since there was no vasopressin in the culture medium, no significant osmotic water permeability in non-transfected cells was expected (Fig. 2E). Cells transfected with mEos2 fluorescent protein alone displayed no significant apical water permeability, confirming that the transfection procedures per se did not confer osmotic water permeability (Fig. 2F). In contrast, transfection with either AQP2-mEos2 or mEos2-AQP2 constructs conferred apical osmotic water permeability to mpkCCD cells (Fig. 2E and 2F), indicating that these chimeric proteins are functional water channels. The exponential time constant $(\tau)$ of calcein fluorescence decay for AQP2-mEos2 and mEos2-AQP2 constructs were $12.8 \pm 0.8 \mathrm{~s}$ ( $n=57$ cells) and $22.6 \pm 1.9 \mathrm{~s}$ ( $\mathrm{n}=39$ cells), respectively. Cells transfected with AQP2-mEos 2 constructs displayed higher apical osmotic water permeability (smaller $\tau$ ) compared to 


\section{Cellular Physiology Cell Physiol Biochem 2015;36:670-682 \begin{tabular}{l|l}
\hline DOI: 10.1159/000430129 & O 2015 S. Karger AG, Basel
\end{tabular} and Biochemistry Published online: May 21, 2015 www.karger.com/cpb}

Yip et al.: Expression of Aquaporin-2 Tagged with a Photoconvertible Protein

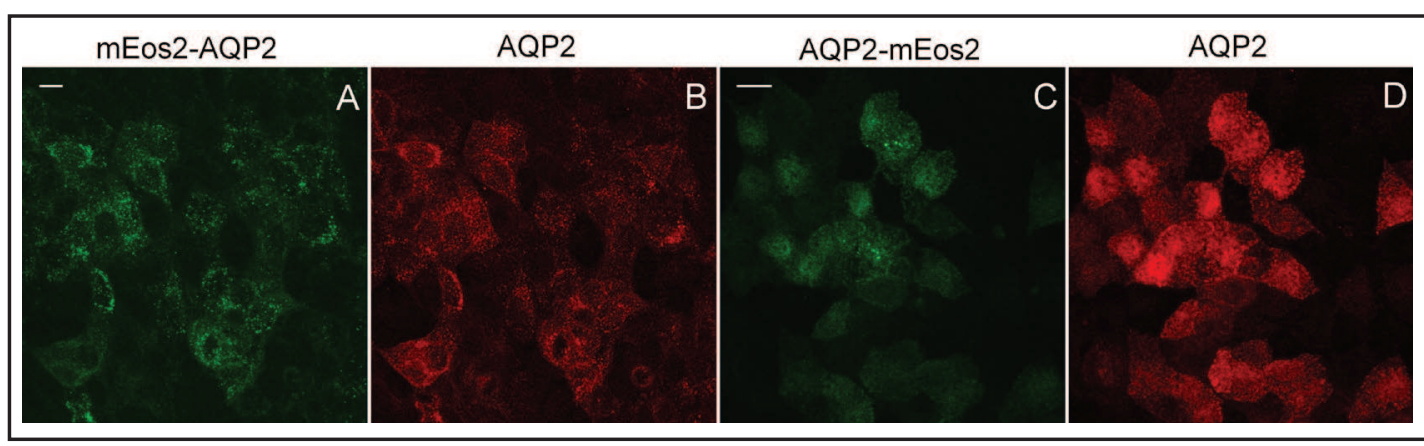

Fig. 1. Colocalization of mEos 2 fluorescence and $A Q P 2$ immunofluorescence in mpkCCD cells grown on permeable filters. mpkCCD cells were transfected with DNA constructs of mEos2-AQP2 (A, B), or AQP2-mEos2 (C, D). Images are projected images of optical sections collected with $0.3 \mu \mathrm{m}$ apart. mEos 2 fluorescence is in green $(A, C)$ and AQP2 immunofluorescence is in red (B, D). These results are representative of five independent transfection experiments. Scale bar is $10 \mu \mathrm{m}$.

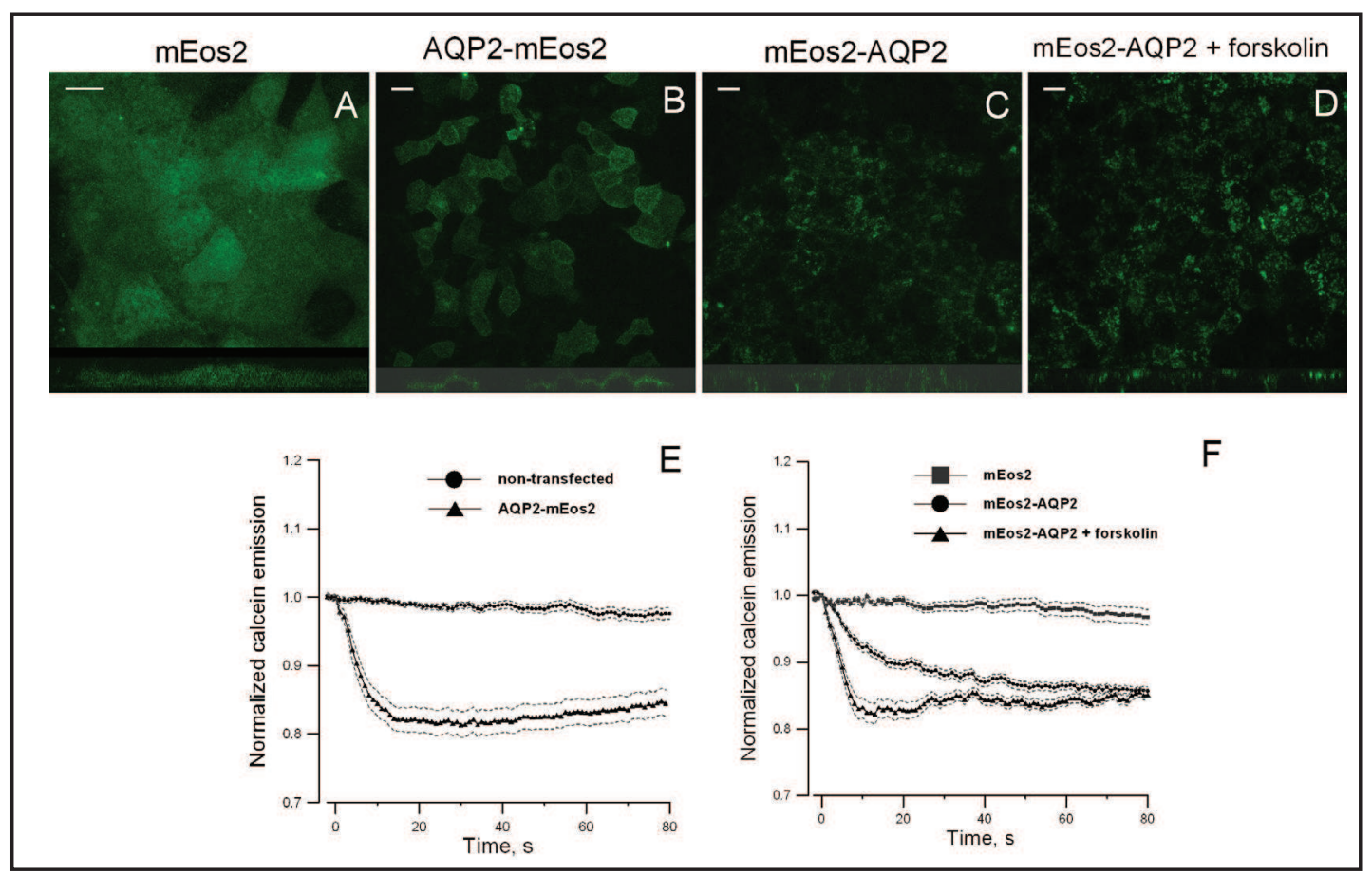

Fig. 2. Localization of mEos 2 and mEos2-tagged AQP2 chimeras expressed in mpkCCD cells grown on permeable filters. mpkCCD cells were transfected with DNA constructs with (A) mEos2, (B) AQP2-mEos2, (C) mEos2-AQP2 and (D) mEos2-AQP2 after 30 min of forskolin stimulation. Images are projected images of optical sections collected with $0.3 \mu \mathrm{m}$ apart from live mpkCCD cells. The lower panel in each panel is the $\mathrm{xz}$-section. These results are representative of five independent transfection experiments. Scale bar is 10 $\mu \mathrm{m}$. The time courses of calcein fluorescence decrease induced by apical hypotonic solution in each type of transfected cells are shown in (E) and (F).The exponential time constant $(\tau)$ of calcein fluorescence decay is $12.8 \pm 0.8 \mathrm{~s}$ ( $\mathrm{n}=57$ cells) in AQP2-mEos2 expressing cells. Forskolin stimulation ( $10 \mu \mathrm{M}, 30 \mathrm{~min}$ ) decreased $\tau$ in mEos2-AQP2 expressing cells from $22.6 \pm 1.9 \mathrm{~s}$ ( $\mathrm{n}=39$ cells) to $10.4 \pm 1.8$. $\mathrm{s}(\mathrm{n}=49$ cells). The apical solution was switched from 290 mOsm buffer to $150 \mathrm{mOsm}$ buffer at time=0. Dotted lines are standard error of the mean. Apical hypotonic solution did not quench calcein fluorescence in non-transfected cells or cells transfected with mEos2 only.

mEos2-AQP2 probably because AQP2-mEos2 chimeric proteins are present constitutively at high density in the apical membrane in non-stimulated condition (Fig. 2B). In cells 


\section{Cellular Physiology Cell Physiol Biochem 2015;36:670-682 \begin{tabular}{l|l}
\hline DOI: 10.1159/000430129 & O 2015 S. Karger AG, Basel
\end{tabular}

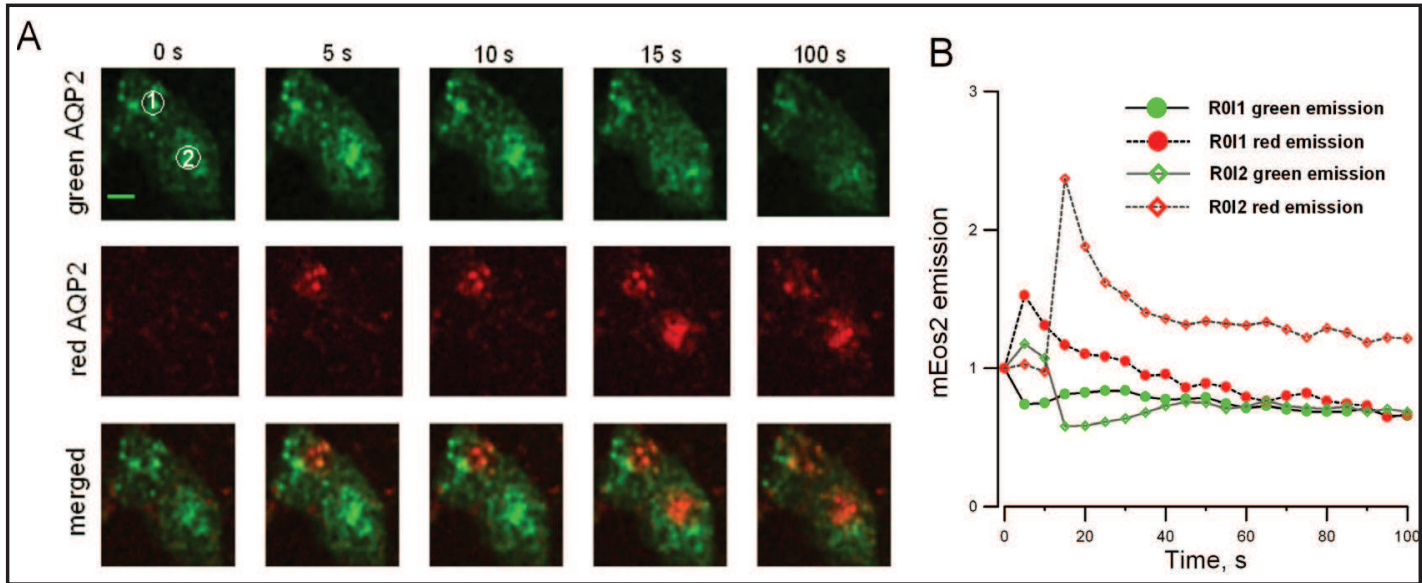

Fig. 3. Photoconversion of mEos2-AQP2 fusion protein expressed in mpkCCD cells. (A) time lapse images of two consecutive local subcellular photoconversions of green mEos2-AQP2 to red mEos2-AQP2, (B) the reciprocal temporal relationship of green and red fluorescence emission in two regions of interest, ROI1 and ROI2 in (A). White circles are the $405 \mathrm{~nm}$ laser irradiation conversion-spots. Scale bar is $5 \mu \mathrm{m}$ in. Cells were grown on collagen-coated glass bottom dish.

transfected with mEos2-AQP2 constructs, the fusion proteins were predominately localized to intracellular vesicles with a minor population present in the apical membrane (Fig. 2C). Forskolin stimulation $(10 \mu \mathrm{M}, 30$ minutes) to increase adenylyl cyclase activity enhanced apical osmotic water permeability of mpkCCD cells transfected with mEos2-AQP2 when compared to non-stimulated cells. The $\tau$ of calcein fluorescence decay was further reduced by

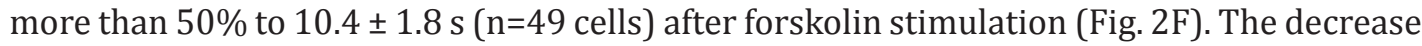
of time constant (increase of water permeability) suggested that forskolin stimulation triggered an accumulation of mEos2-APQ2 on the apical membrane, which was confirmed in optical sections collected from the forskolin stimulated cells (Fig. 2D).

Photoconversion of mEos2-AQP2 chimeric protein in transfected mpkCCD cells

mpkCCD cells transfected with mEos2 DNA exhibited ubiquitous expression of mEos2 protein throughout the cytoplasm (Fig. 2A). In contrast to mEos2, mEos2-AQP2 chimeric proteins were distributed primarily in vesicular compartments. Subcellular local photoactivation of mEos2-AQP2 with a $405 \mathrm{~nm}$ laser pulse ( $0.5 \mathrm{~s})$ resulted in local conversion of green mEos2-AQP2 to red mEos2-AQP2 (Fig. 3A). The time courses of green and red fluorescence emission at two irradiation sites in two mpkCCD cells are also shown (Fig. 3B). A reciprocal temporal relationship of green and red emission was observed immediately after photoconversion at each site. Two discrete populations of colored vesicles could be clearly discerned after photoconversion in each cell. The recovery of green emission and decay in red emission over time were due to the translocation of fluorescent vesicles in and out of the regions of interest, respectively. These observations demonstrated that mEos2 can be used as a photoconvertible fluorescent tag to label AQP2 in mpkCCD cells, and that it is feasible to conduct pulse-chase experiments of two discrete mEos2-AQP2 populations following photoconversion.

Time-lapse monitoring of mEos2-AQP2 vectorial apical trafficking and accumulation in apical membrane

The 3D distribution of AQP2 fusion protein over time was monitored in the reconstructed $3 \mathrm{D}$ images from the optical sections taken every ten minutes. In cells expressing AQP2mEos2, forskolin stimulation $(10 \mu \mathrm{M})$ did not significantly increase the apical accumulation of AQP2-mEos2 over a period of $60 \mathrm{~min}$ (Fig. 4D). The regression coefficients with or without forskolin stimulation were not significantly different. This finding was expected because 


\section{Cellular Physiology Cell Physiol Biochem 2015;36:670-682 \begin{tabular}{l|l}
\hline DOI: 10.1159/000430129 & O 2015 S. Karger AG, Basel
\end{tabular}

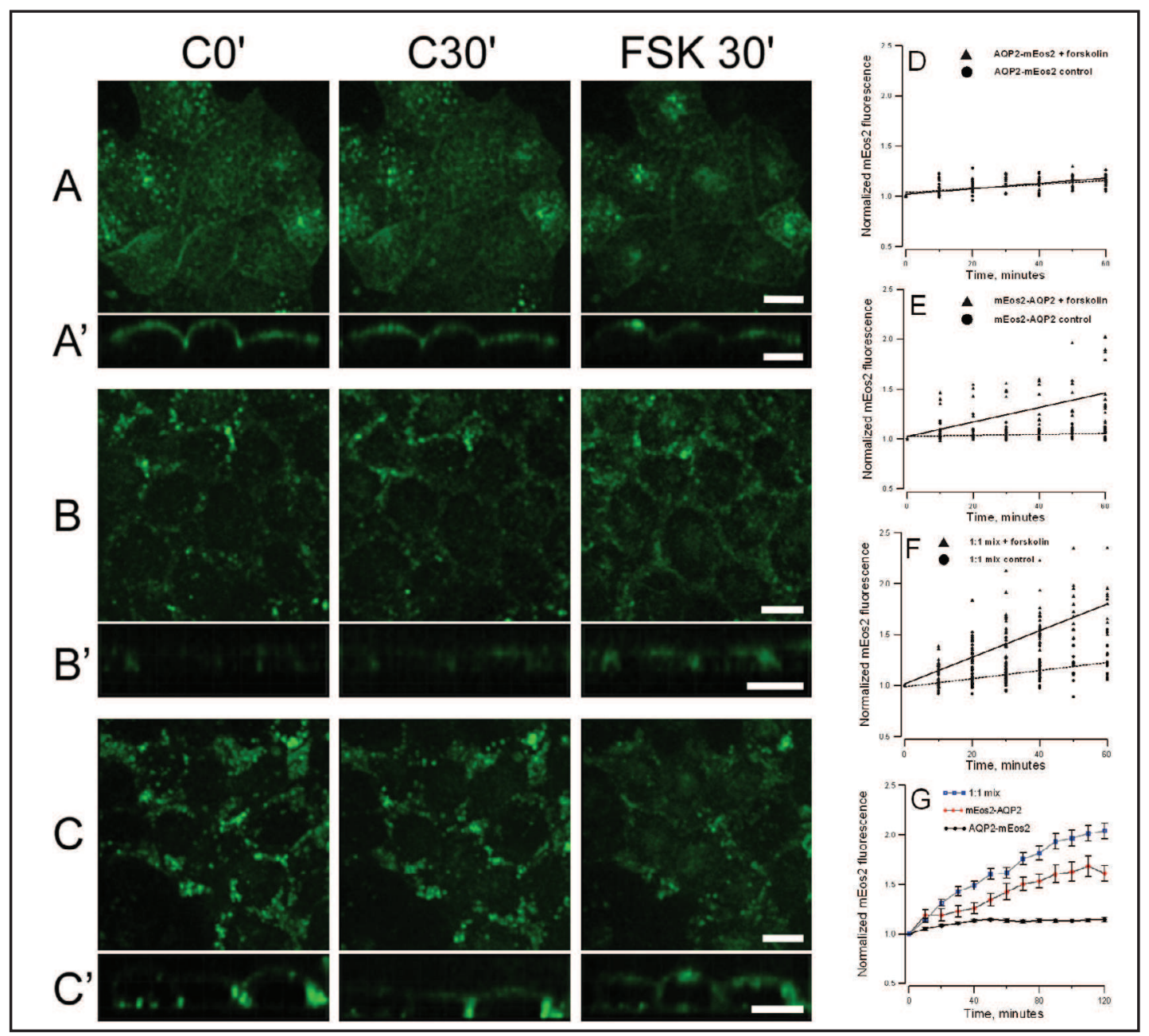

Fig. 4. Time-lapse images of apical trafficking and accumulation of mEo2s fluorescence induced by forskolin in mpkCCD cells grown on collagen-coated glass bottom dish. (A) AQP2-mEos2, (B) mEos2-AQP2, and (C) 1:1 co-transfection of both AQP2-mEos2 and mEos2-AQP2. A', B'. and C' are the xz section views. Each xy image is the projected image of 12 optical sections ( $1 \mu \mathrm{m}$ step). $\mathrm{C}^{\prime}{ }^{\prime}=$ control at time zero, C30' = incubated with buffer for $30 \mathrm{~min}$, FSK30' = incubated with forskolin for $30 \mathrm{~min}$. Scale bar is $10 \mu \mathrm{m}$. Regression analysis of mEos2 fluorescence accumulation are also shown. Straight lines are the regression lines from data collected in the first $60 \mathrm{~min}$. (D) AQP2-mEos2 (regression coefficient are $1.7 \times 10^{-3} \pm 0.5 \times 10^{-3} / \mathrm{min}$ and $3.2 \times 10^{-3}$ $\pm 0.4 \times 10^{-3} / \mathrm{min}$ for control and forskolin stimulation, $\mathrm{n}=10$ cells), (E) mEos2-AQP2 (regression coefficient are $0.2 \times 10^{-4} \pm 0.1 \times 10^{-4} / \mathrm{min}$ and $6.7 \times 10^{-3} \pm 1.5 \times 10^{-3} / \mathrm{min}$ for control and forskolin stimulation, $\mathrm{p}<0.05$, $\mathrm{n}=14$ cells), (F) 1:1 co-transfection (regression coefficient are $4 \times 10^{-3} \pm 0.4 \times 10^{-3} / \mathrm{min}$ and $13.5 \times 10^{-3} \pm 0.8$ $\mathrm{x} 10^{-3} / \mathrm{min}$ for control and forskolin stimulation, $\mathrm{p}<0.05, \mathrm{n}=31$ cells), and (G) Mean normalized time course of mEos2 fluorescence accumulation stimulated by forskolin for two hours. The difference of regression coefficients are significant among all groups $(\mathrm{p}<0.05)$ when stimulated by forskolin.

AQP2-mEos2 fusion proteins were localized constitutively in apical membrane before forskolin stimulation. Fig. 4A shows the apical localization of AQP2-mEos2 in control, $30 \mathrm{~min}$ after incubation in buffer, and $30 \mathrm{~min}$ after forskolin stimulation. Forskolin stimulation did not increase apical accumulation of AQP2-mEos2 significantly, but did trigger AQP2-mEos2 aggregation into apical patches in some cells. In contrast, forskolin stimulation significantly increased the mEos2-AQP2 apical accumulation as compared to the control (Fig. 4B and 4E). The regression coefficient was significantly increased in the presence of forskolin stimulation. 


\section{Cellular Physiology Cell Physiol Biochem 2015;36:670-682 \begin{tabular}{l|l|l}
\hline DOI: 10.1159/000430129 & O 2015 S. Karger AG, Basel
\end{tabular} and Biochemistry Published online: May 21, 2015 www.karger.com/cpb}

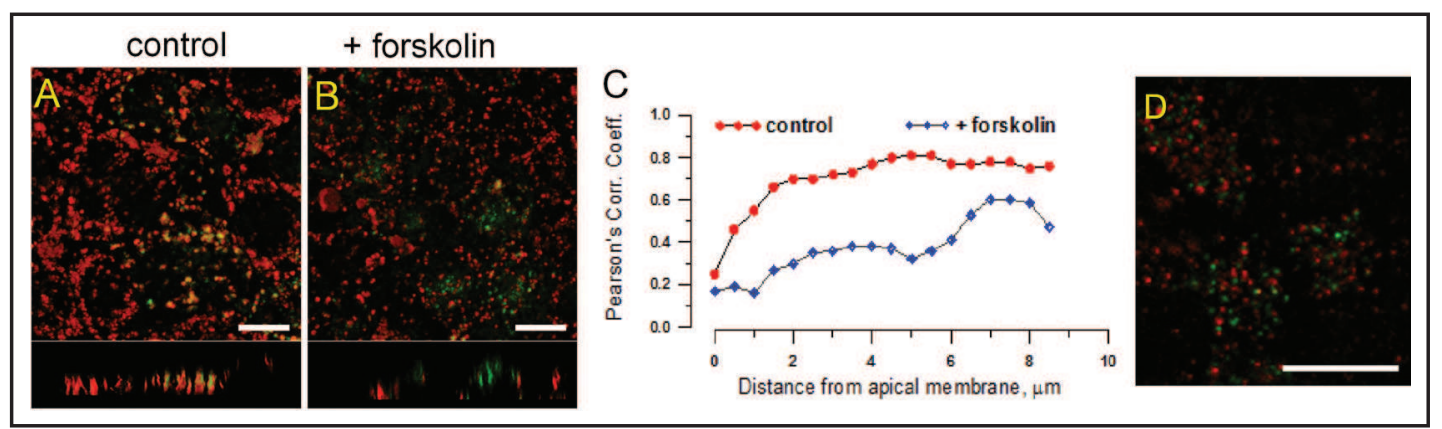

Fig. 5. Localization of mEos2-AQP2 chimeric proteins (green) and acidic organelles (red) of live mpkCCD cells grown on collagen-coated glass bottom dish before (A) and after forskolin stimulation (B). The lower panels are the xz section views of the merged images. The corresponding Pearson's Correlation Coefficients in each xy optical section in (A) and (B) are shown in (C). (D) is the localization of mEos2-AQP2 (green) and immunofluorescence of Lamp-1 (red). Scale bar is $10 \mu \mathrm{m}$.

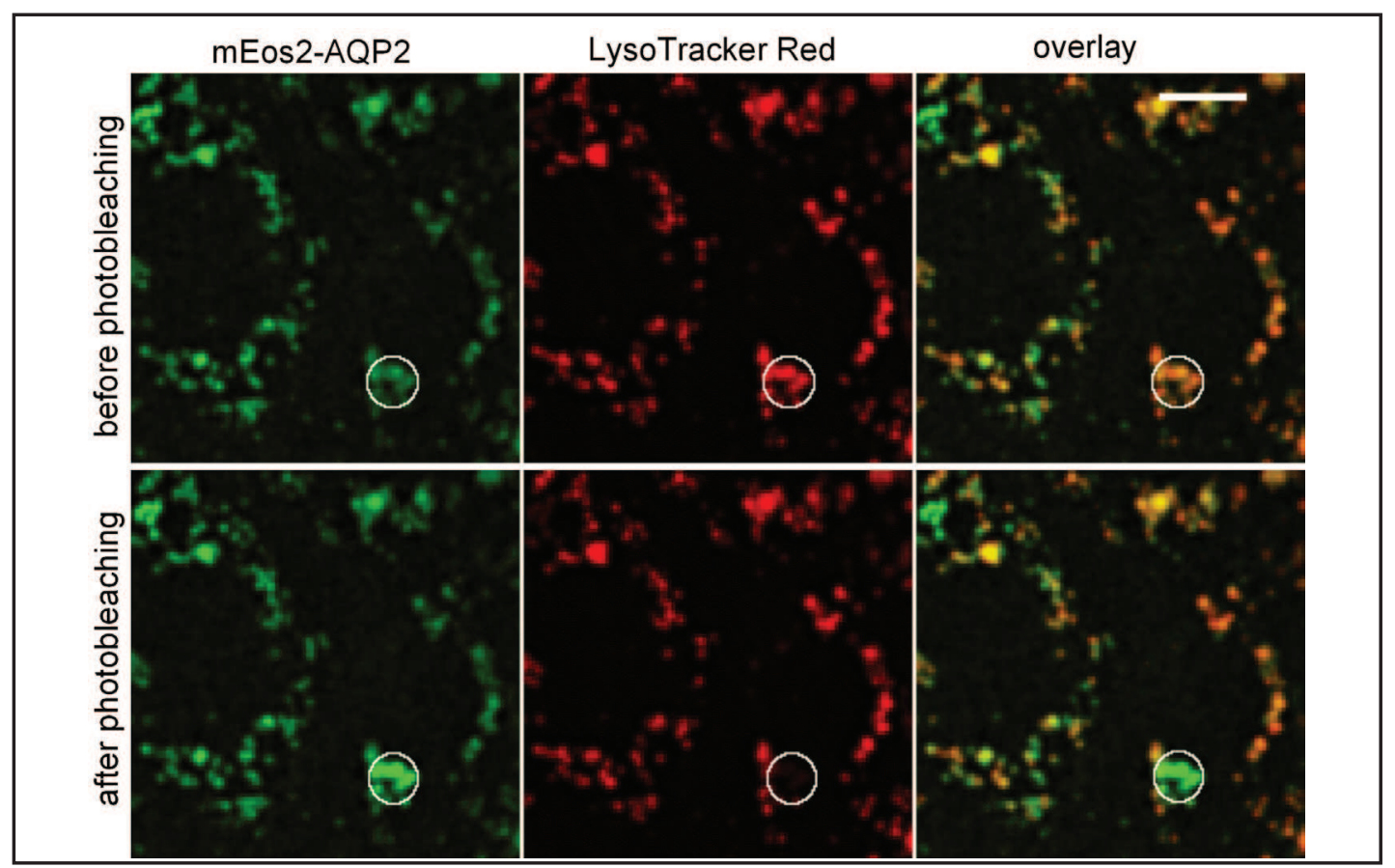

Fig. 6. Colocalization of mEos2-AQP2 chimeric proteins and acidic organelles in live mpkCCD cells grown on collagen-coated glass bottom dish. Acidic organelles were labeled with LysoTracker Red. White circle is the $561 \mathrm{~nm}$ laser irradiation photobleaching spot. After photobleached of LysoTracker Red, the fluorescence intensity of mEos2-AQP2 was enhanced. Förster resonance energy transfer (FRET) was detected between the mEos2-AQP2 (donor) and LysoTracker Red (acceptor). Scale bar is $5 \mu \mathrm{m}$.

These observations were consistent with the measured osmotic water permeability when mEos2-AQP2 expressing cells were stimulated with forskolin on permeable filters (Fig. 2F).

\section{Augmentation of apical trafficking and accumulation in co-transfection}

Co-transfection of AQP2-mEos2 and mEos2-AQP2 constructs at 1:1 ratio $(0.25 \mu \mathrm{g}$ DNA $/ 1 \times 10^{5}$ cells of each DNA construct ) increased the rate of forskolin-induced apical accumulation (regression coefficient) of AQP2 fusion protein by almost two folds when compared to mEos2-AQP2 transfection with the same amount of total DNA (Fig. 4C and $4 \mathrm{~F}$ ). The difference of regression coefficients are significant among all groups with forskolin stimulation $(\mathrm{p}<0.05)$. The time courses of forskolin induced mEos2 fluorescence 


\section{Cellular Physiology Cell Physiol Biochem 2015;36:670-682 \begin{tabular}{l|l}
\hline DOI: 10.1159/000430129 & O 2015 S. Karger AG, Basel
\end{tabular}

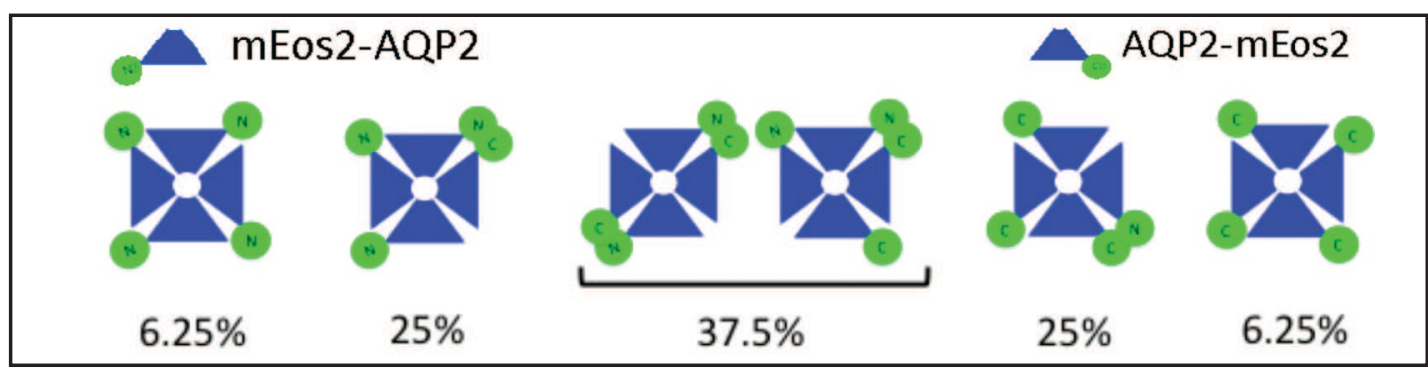

Fig. 7. Six possible configurations of tetrameric aquaporin 2 when AQP2-Eos and Eos-AQP2 constructs are cotransfected at 1:1 ratio. At least one site of close proximity between two mEos proteins is found in all heterotetramers. The percentages are the relative abundance based on binominal distribution.

accumulation in cells transfected with AQP2-mEos2, mEos2-AQP2, and 1:1 co-transfection are shown in Fig. 4G for comparison. The apical accumulation was slowing downing after 60 min.

Localization of mEos2-AQP2 in intracellular acidic compartments

Early endosomes, late endosomes, recycling endosomes, and lysosomes are vesicular compartments which are acidified by vacuolar $\mathrm{H}^{+}$-ATPase to various degrees and collectively make up the endosomal trafficking network. mEos2-AQP2 fusion proteins were expected to be found within these vesicular compartments if there are constitutive endocytosis and recycling of mEos2-AQP2 in mpkCCD cells. To test whether mEos2-AQP2 are present in the acidic vesicular compartments (acidic organelles) in live cells, cells expressing mEos2AQP2 were loaded with LysoTracker Red ( $20 \mathrm{nM}, 15 \mathrm{~min})$ to label the acidic organelles. The fluorescence of mEos2-AQP2 and LysoTracker Red were found to overlap substantially in the baseline condition (Fig. 5A). Forskolin stimulation (10 $\mu \mathrm{M}, 30$ minutes) induced the apical accumulation of mEos2-AQP2 (Fig. 5B), and reduced the colocalization of mEos2AQP2 with LysoTracker Red labeled organelles. The Pearson's correlation coefficient at each xy optical-section as a function of distance from apical membrane toward basolateral membrane in Fig. 5A and 5B are shown in Fig. 5C. Reduced Pearson's correlation coefficient was found in each xy optical section after forskolin stimulation. The mean Pearson's correlation coefficient between mEos2-AQP2 and LysoTracker Red was reduced from 0.54 \pm 0.02 to $0.36 \pm 0.03$ ( 5 series of xyz images from five dishes) after forskolin stimulation. The decrease in Pearson's correlation coefficient suggested that forskolin stimulated mEos2AQP2 to translocate from acidic vesicular storage sites to the apical membrane. When using Lamp-1 as a lysosome marker, there was a partial overlapping of mEos2-AQP2 fluorescence with Lamp-1 immunofluorescence (Fig. 5D), as indicated by the low positive Pearson's correlation coefficient of 0.01 . Compared to extensive overlapping of mEos-AQP2 with LysoTracker Red, it seemed that lysosomes accounted for only a fraction of acidic organelles labeled by LysoTracker Red, and that mEos-AQP2 was not actively directed to lysosomes for degradation. Photobleaching of Lysotracker Red labeled vesicles with $561 \mathrm{~nm}$ laser enhanced the fluorescence of mEos2-AQP2 (Fig. 6), substantiating the notion that mEos2$\mathrm{AQP} 2$ is localized in acidic organelles.

\section{Discussion}

In this study, mEos2 was used as a photoconvertible fluorescent tag to label AQP2 for monitoring the real-time trafficking of AQP2 in mpkCCD cells. Tagging AQP2 with a green fluorescent protein was first introduced by Gustafson et al. in LLC-PK ${ }_{1}$ cells [5], in which only AQP2 with GFP fused to the amino-terminus (GFP-AQP2) displayed cAMP sensitive vesicular trafficking. AQP2 with GFP fused to carboxyl-terminus (AQP2-GFP) was localized constitutively in both apical and basolateral membrane. Both amino- and carboxyl-termini 


\section{Cellular Physiology Cell Physiol Biochem 2015;36:670-682 \\ \begin{tabular}{l|l|l}
\hline DOI: 10.1159/000430129 & O 2015 S. Karger AG, Basel
\end{tabular} \\ \begin{tabular}{l|l} 
and Biochemistry Published online: May 21, 2015 & www.karger.com/cpb
\end{tabular} \\ Yip et al.: Expression of Aquaporin-2 Tagged with a Photoconvertible Protein}

are essential for trafficking of AQP2 to intracellular vesicles and its shuttling to and from the apical membrane [22]. The observations of Gustafson et al. [5] demonstrated that attachment of fluorescent protein in the amino-terminus does not interfere AQP2 trafficking.

AQP2 has four serine phosphorylation sites as well as an ubiquitination site close to the carboxyl terminus. The phosphorylation status of these serine sites determines whether the AQP2 protein will stay on the plasma membrane or will be retrieved by endocytosis to the cytoplasm $[4,23]$. GFP fused in carboxyl-terminus most likely interferes with the regulatory functions of these sites because of the proximity of GFP. Spacers made of several alanine and glutamine residues have been used previously to study subunit arrangement of $\gamma$-aminobutyric acid type A receptors $\left(\mathrm{GABA}_{\mathrm{A}}\right)$. These studies demonstrated that spacer molecules can be used successfully to link subunits together in a multimeric channel without interfering with the function [24]. Spacer of different lengths ranging from 2 to 17 amino acids have also been used to link KillerRed, a fluorescent protein with photosensitizing activity, to aquaporin- 1 and aquaporin- 4 for chromophore-assisted light inactivation studies in live cells [25]. It was demonstrated that the length of the spacer is critical for intracellular chromophore-assisted light activation to inhibit water permeability. The inhibitory effect falls off quickly with increasing the length of the spacer. To test whether the proximity effects of a fluorescent protein in AQP2 carboxyl terminus might be alleviated by introducing a spacer between the AQP2 and the fluorescent tag, a spacer made of 19 amino acids was inserted between mEos2 and AQP2. Our observations indicated the inclusion of a spacer in the carboxyl terminus of AQP2 did not rescue the proximity effects of a fluorescent protein on AQP2 trafficking. AQP2-mEos2 fusion proteins were found to localize constitutively in the apical membrane in mpkCCD cells without any stimulation, which is similar to the localization pattern of expressing AQP2-GFP in LLC-PK 1 cells [5]. These observations confirmed that attachment of a fluorescent protein tag to the carboxyl terminus of AQP2 impairs the normal AQP2 trafficking in both LLC-PK 1 and mpkCCD cells.

On the other hand, the use of a 19 amino acid spacer between the mEos2 and AQP2 amino-terminus preserved the vectorial vesicular trafficking of AQP2 as found in native principle cells of the collecting duct. mEos2-AQP2 chimeras were translocated from vesicular compartments and were accumulated in apical plasma membrane in response to forskolin stimulation. Forskolin stimulated apical accumulation of mEos2-AQP2 was observed when mpkCCD cells were grown on either permeable filters or on collagen-coated glass bottom dishes (Fig. 2 and 4). However, forskolin stimulated GFP-AQP2 translocation to the basolateral membrane in LLC-PK ${ }_{1}$ cells [5]. It is not clear whether this difference is due the use of a 19 amino acid spacer in mEos2-AQP2 or it is a cell line specific phenomenon.

Local photoactivation of mEos2-AQP2 with a $405 \mathrm{~nm}$ laser pulse resulted in local conversion of green mEos2-AQP2 to red mEos2-AQP2. Both green and red mEos2-AQP2 vesicles displayed similar spontaneous non-vectorial movement in the cytoplasm. Two discrete populations of vesicles could be clearly discerned for 2 hours after photoconversion, indicating that the photoconversion is likely to be permanent. The time course of two consecutive photoconversions of mEos2-AQP2 in two regions of interest (ROI) suggested that there were priming effects of the first $405 \mathrm{~nm}$ UV pulse for the subsequent photoconversion (Fig. 3B). The first UV pulse not only triggered photoconversion at ROI1, but also increased the green emission of mEos2-AQP2 at ROI2. The second UV pulse triggered a more intense photoconversion. It is most likely because the first UV laser pulse increased the sensitivity of mEos 2 to $488 \mathrm{~nm}$ excitation. These effects of photoisomerization have been observed in GFP [26].

By exploiting the high temporal resolution of spinning disk confocal microscopy, the apical trafficking and accumulation of mEos2-AQP2 were visualized by acquiring optical section stacks over time. As expected, forskolin stimulation triggered vectorial trafficking and apical accumulation of mEos2-AQP2 but not AQP2-mEos2. Blocking protein synthesis with cycloheximide $(10 \mathrm{mg} / \mathrm{ml})$ before forskolin stimulation did not abolish the apical accumulation of mEos2-AQP2 (data not shown), suggesting that synthesis of mEos2-AQP2 is not necessary to initiate the apical accumulation. However, apical accumulation of mEos2- 


\section{Cellular Physiology Cell Physiol Biochem 2015;36:670-682 \\ \begin{tabular}{l|l} 
DOI: 10.1159/000430129 & O 2015 S. Karger AG, Basel
\end{tabular} \\ and Biochemistry Published online: May 21, $2015 \quad$ www.karger.com/cpb}

AQP2 was barely detected after 10 min of forskolin stimulation, and had not reached the plateau phase after $60 \mathrm{~min}$ of stimulation. On the other hand, vasopressin induced increase apical exocytosis and osmotic water permeability were detected within $40 \mathrm{~s}$ and reached the plateau phase after $30 \mathrm{~min}$ in perfused kidney collecting duct [27-29]. These observations suggested that mEos2-AQP2 retains the vectorial trafficking properties of native AQP2, but the trafficking speed and/or efficiency may be reduced compared to native AQP2 in intact renal collecting duct.

AQP2 is expressed as a tetramer [30]. It has been suggested that the stoichiometry of the phosphorylated and nonphosphorylated subunits might dictate the steady state localization of AQP2 in principal cells [31]. The co-transfection experiment was originally designed to test which AQP2 chimera is dominant in determining the steady state localization of $\mathrm{AQP2}$. Unexpectedly, co-transfection of mEos2-AQP2 and AQP2-mEos2 chimeras at a 1:1 ratio increased the rate of apical accumulation of $\mathrm{AQP} 2$ fusion protein by nearly two fold as compared to mEos2-AQP2 alone (Fig. 4). Assuming equal efficiency in assembly for the two chimeras, calculations using the binominal equation predict that only $6.25 \%$ of the assembled tetrameric water channels are homotetramer of AQP2-mEos2 or mEos2-AQP2 (Fig. 7). Therefore, greater than $87 \%$ of the resulting AQP2 tetramers are heterotetrameric channels with stoichiometry 1:3 (25\%), 2:2 (37.5\%) and 3:1 (25\%) of AQP2-mEos2 and mEos2-AQP2 subunit(s), respectively. The homotetrameric AQP2-mEos2 is expected to localize constitutively in apical membrane as shown in Fig. 2B. Our observations in the cotransfection experiments were consistent with this prediction. Some mEos2 fluorescence was localized to apical membrane before forskolin stimulation (Fig. 4C). However, only $6.25 \%$ of tetramers are homotetramers of mEos2-AQP2 which are known to be responsive to forskolin stimulation by trafficking to the apical membrane. Our observation demonstrated a markedly greater population of the AQP2 chimera trafficked and accumulated following forskolin stimulation in co-transfection than mEos2-AQP2 alone. It has been shown in sodium bicarbonate cotransporter (NBCe1) that a heterodimer complex allows different monomers to structurally complement each other resulting in a dimer with wild-type like function [32]. Thus, it is speculated that heterotetramer formation might be responsible for the increased rate of apical accumulation with forskolin stimulation. Alternatively, the increased apical accumulation of the heterotetramers may be the result of their resistance to endocytotic retrieval in comparison to mEos2-AQP2 homotetramers.

The endosomal trafficking network is composed of vesicular compartments which are acidified by vacuolar $\mathrm{H}^{+}$-ATPase to various degrees. LysoTracker Red is a membrane permeant dye, which is highly selective for acidic organelles. The high value of Pearson's correlation coefficient between mEos2-AQP2 and LysoTracker Red suggested that a significant fraction of mEos2-AQP2 is residing on acidic organelles in mpkCCD cells. These observations are consistent with the studies of expressing non-fluorescent AQP2 in LLC-PK 1 cells and MDCK cells [33-36]. The AQP2 recycling between plasma membrane and cytoplasm is vacuolar $\mathrm{H}^{+}$ ATPase dependent in LLC-PK 1 cells $[35,36]$. AQP2 are partially colocalized with lysosomes, EEA-1 positive early endosomes, and Rab11-positive recycling endosomes in MDCK cells $[33,34]$. Furthermore, loading of LysoTracker Red quenched the emission of mEos2-AQP2, and photobleaching of LysoTracker Red with $561 \mathrm{~nm}$ laser line augmented the emission of mEos2-AQP2 (Fig. 6). These observations implicated that there is Förster resonance energy transfer (FRET) between mEos2-AQP2 and LysoTracker Red, which is a clear indication of colocalization of AQP2 in acidic organelles in live mpkCCD cells. The decrease of mean Pearson's correlation coefficient between mEos2-AQP2 and LysoTracker Red after forskolin stimulation suggested translocation of mEos2-AQP2 out of the acidic organelles into the apical membrane. The distribution of Pearson's correlation coefficient across the cells revealed a similar relationship. In the basal condition, the coefficients close to basolateral membrane were high but decreased rapidly when approaching the apical membrane (Fig. 5). Forskolin stimulation not only reduced the coefficient in every xy optical section but also the gradient of Pearson's correlation coefficient across the cells. 


\section{Cellular Physiology Cell Physiol Biochem 2015;36:670-682 \begin{tabular}{c|c|c|c|}
\hline DOI: 10.1159/000430129 & (c) 2015 S. Karger AG, Basel
\end{tabular} \begin{tabular}{l|l} 
and Biochemistry Published online: May 21, 2015 & www.karger.com/cpb \\
\hline
\end{tabular}}

Yip et al.: Expression of Aquaporin-2 Tagged with a Photoconvertible Protein

In conclusion, our observations demonstrate that mEos2 can be used as a photoconvertible fluorescent tag to label AQP2 in mpkCCD cells, which allows the trafficking of two discrete populations of AQP2 containing vesicles to be monitored simultaneously. The $\mathrm{mEos} 2-\mathrm{AQP} 2$ fusion proteins are residing in acidic organelles before forskolin stimulation, and mEos2-AQP2 preserves the vectorial vesicular trafficking of AQP2 as found in native principle cells. By serendipity in experimental design, the results also suggest that formation of heterotetramers of AQP2 with mEos2 tagged in the carboxyl- and amino-terminus increase the rate of apical accumulation of the chimeric protein.

\section{Acknowledgements}

This study was supported by a Grant-In-Aid from the American Heart Association Greater Southeast to K.P.Yip.

\section{Disclosure Statement}

Nothing to disclose.

\section{References}

1 Strait KA, Stricklett PK, Chapman M, Kohan DE: Characterization of vasopressin-responsive collecting duct adenylyl cyclases in the mouse. Am J Physiol Renal Physiol 2010;298:F859-867.

2 Nielsen S, Chou CL, Marples D, Christensen EI, Kishore BK, Knepper MA: Vasopressin increases water permeability of kidney collecting duct by inducing translocation of aquaporin-CD water channels to plasma membrane. Proc Natl Acad Sci U S A 1995;92:1013-1017.

3 Nielsen S, Frokiaer J, Marples D, Kwon TH, Agre P, Knepper MA: Aquaporins in the kidney: from molecules to medicine. Physiol Rev 2002;82:205-244.

4 Moeller HB, Praetorius J, Rutzler MR, Fenton RA: Phosphorylation of aquaporin-2 regulates its endocytosis and protein-protein interactions. Proc Natl Acad Sci U S A 2010;107:424-429.

5 Gustafson CE, Levine S, Katsura T, McLaughlin M, Aleixo MD, Tamarappoo BK, Verkman AS, Brown D: Vasopressin regulated trafficking of a green fluorescent protein-aquaporin 2 chimera in LLC-PK1 cells. Histochem Cell Biol 1998;110:377-386.

6 Noda Y, Horikawa S, Kanda E, Yamashita M, Meng H, Eto K, Li Y, Kuwahara M, Hirai K, Pack C, Kinjo M, Okabe S, Sasaki S: Reciprocal interaction with G-actin and tropomyosin is essential for aquaporin-2 trafficking. J Cell Biol 2008;182:587-601.

7 Chudakov DM, Matz MV, Lukyanov S, Lukyanov KA: Fluorescent proteins and their applications in imaging living cells and tissues. Physiol Rev 2010;90:1103-1163.

8 Shroff H, Galbraith CG, Galbraith JA, Betzig E: Live-cell photoactivated localization microscopy of nanoscale adhesion dynamics. Nat Methods 2008;5:417-423.

9 Yeh YC, Wu CC, Wang YK, Tang MJ: DDR1 triggers epithelial cell differentiation by promoting cell adhesion through stabilization of E-cadherin. Mol Biol Cell 2011;22:940-953.

10 McKinney SA, Murphy CS, Hazelwood KL, Davidson MW, Looger LL: A bright and photostable photoconvertible fluorescent protein. Nat Methods 2009;6:131-133.

11 Sandoval PC, Slentz DH, Pisitkun T, Saeed F, Hoffert JD, Knepper MA: Proteome-wide measurement of protein half-lives and translation rates in vasopressin-sensitive collecting duct cells. J Am Soc Nephrol 2013;24:1793-1805.

12 Bolger SJ, Hurtado PA, Hoffert JD, Saeed F, Pisitkun T, Knepper MA: Quantitative phosphoproteomics in nuclei of vasopressin-sensitive renal collecting duct cells. Am J Physiol Cell Physiol 2012;303:C1006-1020.

13 Schenk LK, Bolger SJ, Luginbuhl K, Gonzales PA, Rinschen MM, Yu MJ, Hoffert JD, Pisitkun T, Knepper MA: Quantitative proteomics identifies vasopressin-responsive nuclear proteins in collecting duct cells. J Am Soc Nephrol 2012;23:1008-1018. 


\section{Cellular Physiology Cell Physiol Biochem 2015;36:670-682 \begin{tabular}{l|l}
\hline DOI: 10.1159/000430129 & (c) 2015 S. Karger AG, Base
\end{tabular} \begin{tabular}{l|l} 
and Biochemistry Published online: May 21, 2015 & www.karger.com/cpb \\
\hline
\end{tabular}}

Yip et al.: Expression of Aquaporin-2 Tagged with a Photoconvertible Protein

14 Rinschen MM, Yu MJ, Wang G, Boja ES, Hoffert JD, Pisitkun T, Knepper MA: Quantitative phosphoproteomic analysis reveals vasopressin V2-receptor-dependent signaling pathways in renal collecting duct cells. Proc Natl Acad Sci U S A 2010;107:3882-3887.

15 Hasler U, Mordasini D, Bens M, Bianchi M, Cluzeaud F, Rousselot M, Vandewalle A, Feraille E, Martin PY: Long term regulation of aquaporin-2 expression in vasopressin-responsive renal collecting duct principal cells. J Biol Chem 2002;277:10379-10386.

16 Loo CS, Chen CW, Wang PJ, Chen PY, Lin SY, Khoo KH, Fenton RA, Knepper MA, Yu MJ: Quantitative apical membrane proteomics reveals vasopressin-induced actin dynamics in collecting duct cells. Proc Natl Acad Sci U S A 2013;110:17119-17124.

17 van Beest M, Robben JH, Savelkoul PJ, Hendriks G, Devonald MA, Konings IB, Lagendijk AK, Karet F, Deen PM: Polarisation, key to good localisation. Biochim Biophys Acta 2006;1758:1126-1133.

18 Zelenina M, Brismar H: Osmotic water permeability measurements using confocal laser scanning microscopy. Eur Biophys J 2000;29:165-171.

19 Fenton RA, Moeller HB, Nielsen S, de Groot BL, Rutzler M: A plate reader-based method for cell water permeability measurement. Am J Physiol Renal Physiol 2010;298:F224-230.

20 Peng H, Bria A, Zhou Z, Iannello G, Long F: Extensible visualization and analysis for multidimensional images using Vaa3D. Nat Protoc 2014;9:193-208.

21 Peng H, Ruan Z, Long F, Simpson JH, Myers EW: V3D enables real-time 3D visualization and quantitative analysis of large-scale biological image data sets. Nat Biotechnol 2010;28:348-353.

22 van Balkom BW, Graat MP, van Raak M, Hofman E, van der Sluijs P, Deen PM: Role of cytoplasmic termini in sorting and shuttling of the aquaporin-2 water channel. Am J Physiol Cell Physiol 2004;286:C372-379.

23 Kuwahara M, Asai T, Terada Y, Sasaki S: The C-terminal tail of aquaporin-2 determines apical trafficking. Kidney Int 2005;68:1999-2009.

24 Baumann SW, Baur R, Sigel E: Subunit arrangement of gamma-aminobutyric acid type A receptors. J Biol Chem 2001;276:36275-36280.

25 Baumgart F, Rossi A, Verkman AS: Light inactivation of water transport and protein-protein interactions of aquaporin-Killer Red chimeras. J Gen Physiol 2012;139:83-91.

26 Brejc K, Sixma TK, Kitts PA, Kain SR, Tsien RY, Ormo M, Remington SJ: Structural basis for dual excitation and photoisomerization of the Aequorea victoria green fluorescent protein. Proc Natl Acad Sci U S A 1997;94:2306-2311.

27 Wall SM, Han JS, Chou CL, Knepper MA: Kinetics of urea and water permeability activation by vasopressin in rat terminal IMCD. Am J Physiol 1992;262:F989-998.

28 Yip KP: Coupling of vasopressin-induced intracellular $\mathrm{Ca}(2+)$ mobilization and apical exocytosis in perfused rat kidney collecting duct. J Physiol 2002;538:891-899.

29 Yip KP: Epac-mediated Ca(2+) mobilization and exocytosis in inner medullary collecting duct. Am J Physiol Renal Physiol 2006;291:F882-890.

30 Verkman AS, Mitra AK: Structure and function of aquaporin water channels. Am J Physiol Renal Physiol 2000;278:F13-28.

31 Kamsteeg EJ, Heijnen I, van Os CH, Deen PM: The subcellular localization of an aquaporin-2 tetramer depends on the stoichiometry of phosphorylated and nonphosphorylated monomers. J Cell Biol 2000;151:919-930.

32 Chang MH, Chen AP, Romero MF: NBCe1A dimer assemble visualized by bimolecular fluorescence complementation (BiFC). Am J Physiol Renal Physiol 2014;F672-F680.

33 Tajika Y, Matsuzaki T, Suzuki T, Ablimit A, Aoki T, Hagiwara H, Kuwahara M, Sasaki S, Takata K: Differential regulation of AQP2 trafficking in endosomes by microtubules and actin filaments. Histochem Cell Biol 2005;124:1-12.

34 Tajika Y, Matsuzaki T, Suzuki T, Aoki T, Hagiwara H, Kuwahara M, Sasaki S, Takata K: Aquaporin-2 is retrieved to the apical storage compartment via early endosomes and phosphatidylinositol 3-kinasedependent pathway. Endocrinology 2004;145:4375-4383.

35 Gustafson CE, Katsura T, McKee M, Bouley R, Casanova JE, Brown D: Recycling of AQP2 occurs through a temperature- and bafilomycin- sensitive trans-Golgi-associated compartment. Am J Physiol Renal Physiol 2000;278:F317-326.

36 Katsura T, Ausiello DA, Brown D: Direct demonstration of aquaporin-2 water channel recycling in stably transfected LLC-PK1 epithelial cells. Am J Physiol 1996;270:F548-553. 